\title{
Lung Ultrasound in COVID-19: Clinical Correlates and Comparison with Chest Computed Tomography
}

\author{
Grazia Portale ${ }^{1}$ (D) Federica Ciolina ${ }^{2}$ - Luca Arcari ${ }^{3} \cdot$ Gianluca Di Lazzaro Giraldi $^{1} \cdot$ Massimiliano Danti $^{2}$. \\ Lorenzo Pietropaolo ${ }^{1}$ - Giovanni Camastra ${ }^{3}$. Chiara Cordischi ${ }^{1}$ - Laura Urbani ${ }^{1}$ - Lidia Proietti ${ }^{1}$ - Luca Cacciotti $^{3}$. \\ Claudio Santini ${ }^{4} \cdot$ Serena Melandri ${ }^{4} \cdot$ Gerardo Ansalone $^{3} \cdot$ Stefano Sbarbati $^{3} \cdot$ Cinzia Sighieri $^{1}$
}

Accepted: 1 June 2021 / Published online: 18 June 2021

(C) The Author(s), under exclusive licence to Springer Nature Switzerland AG 2021

\begin{abstract}
Lung ultrasound (LUS) and chest computed tomography (chest CT) are largely employed to evaluate coronavirus disease 2019 (COVID-19) pneumonia. We investigated semi-quantitative LUS and CT scoring in hospitalized COVID-19 patients. LUS and chest $\mathrm{CT}$ were performed within $24 \mathrm{~h}$ upon admission. Both were analyzed according to semi-quantitative scoring systems. Subgroups were identified according to median LUS score. Patients within higher LUS score group were older (79 vs 60 years, $\mathrm{p}<0.001)$, had higher C-reactive protein (CRP) $(7.2 \mathrm{mg} / \mathrm{dl} \mathrm{vs} 1.3 \mathrm{mg} / \mathrm{dl}, \mathrm{p}<0.001)$ and chest CT score (10 vs 4, $\mathrm{p}=0.027)$ as well as lower $\mathrm{PaO} 2 / \mathrm{FiO} 2$ (286 vs 356, $\mathrm{p}=0.029$ ) as compared to patients within lower scores. We found a significant correlation between scores $(\mathrm{r}=0.390, \mathrm{p}=0.023)$. Both LUS and CT scores correlated directly with patients age $(\mathrm{r}=0.586, \mathrm{p}<0.001$ and $\mathrm{r}=0.399$, $\mathrm{p}=0.021$ respectively) and CRP $(\mathrm{r}=0.472, \mathrm{p}=0.002$ and $\mathrm{r}=0.518, \mathrm{p}=0.002$ respectively), inversely with $\mathrm{PaO} 2 / \mathrm{FiO} 2(\mathrm{r}=-0.485$, $\mathrm{p}=0.003$ and $\mathrm{r}=-0.440, \mathrm{p}=0.017$ respectively). LUS score only showed significant correlation with hs-troponin $\mathrm{T}$, NT-pro-BNP, and creatinine $(r=0.433, p=0.019 ; \mathrm{r}=0.411, \mathrm{p}=0.027$, and $\mathrm{r}=0.497, \mathrm{p}=0.001$, respectively). Semi-quantitative bedside LUS is related to the severity of COVID-19 pneumonia similarly to chest CT. Correlation of LUS score with markers of cardiac and renal injury suggests that LUS might contribute to a more comprehensive evaluation of this heterogeneous population.
\end{abstract}

Keywords Lung ultrasound $\cdot$ Chest CT $\cdot$ COVID-19 pneumonia $\cdot$ Scoring system

\section{Introduction}

In the late 2019, the outbreak in China of a novel type of betacoronavirus 2019-nCov, later renamed severe acute

Grazia Portale and Federica Ciolina equally contributed to the manuscript and should be considered as co-first author

This article is part of the Topical Collection on COVID-19

Grazia Portale

gportale69@gmail.com

1 Emergency Medicine Unit, Madre Giuseppina Vannini Hospital, Via di Acqua Bullicante 4, 00177 Rome, Italy

2 Radiology Unit, Madre Giuseppina Vannini Hospital, Via di Acqua Bullicante 4, 00177 Rome, Italy

3 Covid-Cardiology Unit, Madre Giuseppina Vannini Hospital, Rome, Italy

4 Internal Medicine Department, Madre Giuseppina Vannini Hospital, Rome, Italy respiratory syndrome coronavirus 2 (SARS-CoV-2), spreads to generate a global pandemic of the so-called coronavirus disease 2019 (COVID-19) [1]. This is a respiratory tract infection that may lead to severe systemic involvement with interstitial pneumonia and respiratory failure often associated with myocardial injury [2, 3], thrombosis [4], multiorgan failure, and death [5]. Mainstay of COVID-19 diagnosis is nasopharyngeal swab and subsequent quantitative real-time reverse transcriptase polymerase chain reaction (qRT-PCR) [5], but a central role has been demonstrated for chest computed tomography (chest $\mathrm{CT}$ ) too. Indeed, this can provide differential diagnosis and assess complications [6], while the possibility of detecting COVID-19 pneumonia using a chest CT-only approach has been explored [7]. Lung ultrasound (LUS) is another technique that can be used for lung evaluation in COVID-19 patients, with an expanding role favored by the possibility of performing it bedside [8]. The aim of the present study was to investigate semi-quantitative assessment of LUS and CT findings in a cohort of patients hospitalized for COVID-19 pneumonia, providing comparison with clinical and laboratory data. 


\section{Methods}

\section{Patients' Enrollment}

This is a partly retrospective, observational, single-center study on hospitalized patients with COVID-19 pneumonia. Diagnostic work-up and clinical management of COVID-19 patients at our institution have been previously described in detail [9]. For the purpose of the present study, we included in the analysis patients $(n=42)$ who underwent timely LUS within $24 \mathrm{~h}$ upon hospital admission. All patients included in the analysis had established diagnosis of SARS-CoV-2 infection (qRT-PCR) with pulmonary involvement (chest CT) and were treated in dedicated "COVID units." Past clinical history and symptoms were registered by the accepting physician. Within $24 \mathrm{~h}$ upon admission, all patients underwent routine venous and arterial blood gas (ABG) examination as well as 12-lead ECG. On arterial blood gas test, arterial oxygen concentration ( $\mathrm{PaO} 2)$ was normalized to the fractional volume of the inspired oxygen to calculate the $\mathrm{PaO} 2 / \mathrm{FIO} 2$ ratio.

\section{Chest Computed Tomography (CCT)}

Two multidetector CT scanners (Philips Brilliance 16 and Brilliance 64) were used for all examinations. Scanning parameters were the same as the manufacturer's standard recommended pre-setting for a thorax routine. Images were acquired with a 1-mm slice thickness and a reconstruction increment of $0.5 \mathrm{~mm}$ in all cases using a soft tissue kernel of B20 and a lung kernel of B60. Coronal and sagittal multiplanar reconstructions (MPR) were also done in all cases.

Infection prevention and control measures were guaranteed in all suspected CT cases (sanitation of CT room and patient's isolation). Suspicion of SARS-CoV-2 pneumonia was established by the presence of three CT patterns: ground glass opacity (GGO), crazy paving, and consolidation according to previous publications $[10,11]$.

The semi-quantitative severity score proposed by Pan was used per each of the 5 lobes considering the degree of anatomical involvement [12]. Specifically, we assigned points:

- $\quad 0$, no involvement

- $1,<5 \%$ involvement

- 2, 5-25\% involvement

- $\quad 3,26-50 \%$ involvement

- $\quad 4,51-75 \%$ involvement

- $5,>75 \%$ involvement

The resulting global CT score was the sum of each individual lobar score and (0 to 25). Collateral features such as fibrosis, subpleural lines, pleural and pericardial effusion, and lymphadenopathy were also depicted. Distribution of lung abnormalities was also classified as predominantly subpleural, centrolobular, random (without predilection for subpleural or central regions), or diffuse (continuous involvement without respect to lung segments). Blinded independent image analysis was performed by two radiologists (M.D. and F.C., respectively, with $>20$ and $>10$ years of experience in thoracic radiology) with use of the institutional digital database system (Impax Client, Agfa, version 6.6.0.145, Belgium). Any disagreement was resolved by consensus.

\section{Lung Ultrasound}

Portable ultrasound machines dedicated to exclusive use for patients with COVID-19, both equipped with a 3-6-MHz convex array transducer, were used (GE Vivid $\mathrm{i}$ and Siemens P500). All examinations were performed bedside within $24 \mathrm{~h}$ upon hospital admission. Images were recorded, stored, and analyzed offline by an experienced operator blinded to patients' clinical data and CT findings.

All subjects underwent bedside US scanning and were systematically studied as suggested by Soldati et al. in 14 areas: 3 posterior, 2 lateral, and 2 anterior on each chest side [13]. A score was assigned to each segment according to ultrasonographic appearances of chest US as follows: (1) presence of horizontal artifact, A-lines pattern, with continuous and regular pleural line (score 0); (2) indented pleural with vertical artifacts appearing (score 1); (3) small-to-large consolidated areas with associated white areas (white lung) (score 2); and (4) dense and largely extended white lung with or without larger consolidations (score 3). Finally, a total score in each patient was calculated by summation of values recorded in all 14 segments. Groups with low and high LUS score, respectively, were identified according to median value and comparison provided.

\section{Statistical Analysis}

All analysis was performed using SPSS software 25 (SPSS Inc., Chicago, IL). Data are presented as mean \pm standard deviation, counts (percentages), or median (interquartile range, IQR), as appropriate. Comparisons between groups were performed using Chi-squared test, Fisher's exact test, and Student's t-test for independent samples or Mann-Whitney $\mathrm{U}$ test as appropriate. Analysis of relationships was performed using linear regression analysis and bivariate correlation with corresponding Pearson's or Spearman correlation coefficients as appropriate according to data distribution. Log-transformed values for serum biomarkers (hs-troponin, NT-pro-BNP, Ddimer, CRP, creatinine) were used to yield approximate normality when performing correlation analysis. All tests were two-tailed, and $p$ value of $<0.05$ was considered statistically significant. All patients provided informed consent for the use of their record for research purposes; the study complied with the content of the Declaration of Helsinki. 


\section{Results}

\section{Baseline Characteristics}

Baseline clinical and demographic characteristics in our sample are summarized in Table 1. Mean age was 70 years, and
$48 \%$ were male. Comorbidities were largely prevalent within the study population, where more than half of the patients had known hypertension, approximately one-third had preexisting CVD, and 57\% were taking an ace-inhibitor or angiotensin II receptor blocker before hospital admission. Median CRP and D-dimer were increased (4.6 and 892

Table 1 Baseline demographic and clinical characteristics of the study population overall and stratified by median LUS score value

\begin{tabular}{|c|c|c|c|c|}
\hline Variable & Overall $(n=42)$ & Low LUS score $(n=21)$ & High LUS score $(n=21)$ & $\mathrm{p}$ \\
\hline Age (years) & $70 \pm 18$ & $60 \pm 19$ & $79 \pm 11$ & $<0.001$ \\
\hline Sex (male) & $20(48 \%)$ & $11(55 \%)$ & $9(45 \%)$ & 0.537 \\
\hline \multicolumn{5}{|c|}{ Signs and symptoms at presentation } \\
\hline Cough & $20(48 \%)$ & $10(48 \%)$ & $10(48 \%)$ & 0.591 \\
\hline Dyspnea & $15(36 \%)$ & $5(25 \%)$ & $10(48 \%)$ & 0.204 \\
\hline Fever & $28(66 \%)$ & $13(60 \%)$ & $15(71 \%)$ & $>0.99$ \\
\hline \multicolumn{5}{|l|}{ Coexistent conditions } \\
\hline Hypertension & $29(\%)$ & $11(55 \%)$ & $18(82 \%)$ & 0.145 \\
\hline Dyslipidemia & $8(19 \%)$ & $2(10 \%)$ & $6(27 \%)$ & 0.258 \\
\hline Diabetes & $4(9 \%)$ & $1(4 \%)$ & $3(15 \%)$ & 0.613 \\
\hline Previous CVD & $14(33 \%)$ & $5(25 \%)$ & $9(41 \%)$ & 0.275 \\
\hline - Atrial fibrillation & $8(19 \%)$ & $3(15 \%)$ & $5(23 \%)$ & 0.709 \\
\hline - Coronary artery Disease & $3(7 \%)$ & $3(15 \%)$ & $0(0 \%)$ & 0.083 \\
\hline - Heart failure & $9(22 \%)$ & $3(15 \%)$ & $6(28 \%)$ & 0.476 \\
\hline - Stroke & $3(7 \%)$ & $1(4 \%)$ & $2(10 \%)$ & $>0.99$ \\
\hline CKD & $3(7 \%)$ & $0(0 \%)$ & $3(15 \%)$ & 0.238 \\
\hline COPD & $11(26 \%)$ & $7(35 \%)$ & $4(18 \%)$ & 0.173 \\
\hline Cancer & $4(9 \%)$ & $3(15 \%)$ & $1(4 \%)$ & 0.333 \\
\hline Ace-inhibitor therapy & $24(57 \%)$ & $13(60 \%)$ & $11(55 \%)$ & 0.743 \\
\hline \multicolumn{5}{|l|}{ Laboratory tests } \\
\hline $\mathrm{Hb}(\mathrm{g} / \mathrm{dl})$ & $12.4 \pm 2.5$ & $12.9 \pm 2.6$ & $12 \pm 2.4$ & 0.289 \\
\hline WBC $($ per $\mu \mathrm{l})$ & $7.1(5,9.4)$ & $6(4.3,9.3)$ & $7.5(5.3,10.2)$ & 0.411 \\
\hline Neutrophil (per $\mu \mathrm{l})$ & $4.7(3.2,7.2)$ & $4.2(3.1,7.4)$ & $5.9(3.5,7.3)$ & 0.240 \\
\hline Lymphocyte (per $\mu \mathrm{l})$ & $1.1(0.9,1.6)$ & $1.2(0.99,1.7)$ & $1(0.8,1.5)$ & 0.299 \\
\hline NLR & $4.2(2.3,7.1)$ & $3.4(2,6)$ & $5(2.6,8)$ & 0.147 \\
\hline Creatinine (mg/dl) & $0.8(0.6,1.14)$ & $0.72(0.56,0.94)$ & $1(0.64,1.6)$ & 0.014 \\
\hline CRP (mg/dl) & $4.6(1,7.7)$ & $1.3(0.3,5)$ & $7.2(4.3,13)$ & $<0.001$ \\
\hline D-dimer (FEU) & $892(548,1376)$ & $701(276,2168)$ & $971(745,1216)$ & 0.647 \\
\hline Hs-troponin T (pg/ml) & $17(7,43)$ & $10(6,23)$ & $24(9,62)$ & 0.123 \\
\hline NT-pro-BNP (pg/ml) & $436(85,4171)$ & $187(42,610)$ & $1068(199,1216)$ & 0.057 \\
\hline \multicolumn{5}{|l|}{ Blood gas analysis } \\
\hline $\mathrm{pH}$ & $7.48 \pm 0.08$ & $7.5 \pm 0.1$ & $7.46 \pm 0.05$ & 0.170 \\
\hline pO2 (mmHg) & $73 \pm 16$ & $79 \pm 13$ & $69 \pm 16$ & 0.04 \\
\hline pCO2 (mmHg) & $35 \pm 7$ & $35 \pm 6$ & $34 \pm 7$ & 0.771 \\
\hline $\mathrm{PaO} 2 / \mathrm{FiO} 2$ & $318 \pm 95$ & $356 \pm 97$ & $286 \pm 83$ & 0.029 \\
\hline \multicolumn{5}{|l|}{ Chest CT } \\
\hline CT score (available in $34 / 42$ ) & $7(3,11)$ & $4(2,8)$ & $10(7,13)$ & 0.027 \\
\hline \multicolumn{5}{|l|}{ LUS } \\
\hline LUS score & $6(3,10)$ & $3(1,5)$ & $10(6,14)$ & $<0.001$ \\
\hline
\end{tabular}

LUS lung ultrasound, $C V D$ cardiovascular disease, $C R P$ C-reactive protein, $F E U$ fibrinogen equivalent unit, $C K D$ chronic kidney disease, $C O P D$ chronic obstructive pulmonary disease, $W B C$ white blood cells, $N L R$ neutrophil to lymphocyte ratio, $C T$ computed tomography 
fibrinogen equivalent unit, respectively). Blood gas analysis showed mean $\mathrm{PaO} 2$ of $73 \pm 16 \mathrm{mmHg}$ and mean $\mathrm{PaO} 2 / \mathrm{FIO} 2$ of $318 \pm 95$. Median LUS score was $6(3,10)$, and median CT score was $7(3,11)$.

\section{Characteristics of Population According to LUS Findings}

On subgroups analysis, patients who had higher LUS score were older ( 79 vs 60 years, $\mathrm{p}<0.001$ ) with similar prevalence of CVD (41\% vs 25\%, p=0.275) and other comorbidities, whereas blood examinations showed higher CRP and creatinine $(7.2 \mathrm{mg} / \mathrm{dl}$ vs $1.3 \mathrm{mg} / \mathrm{dl}, \mathrm{p}>0.001$ and $1 \mathrm{mg} / \mathrm{dl}$ vs $0.72 \mathrm{mg} /$ $\mathrm{dl}, \mathrm{p}=0.014$, respectively) and lower $\mathrm{PaO} 2 / \mathrm{FiO} 2$ (286 vs 356, $\mathrm{p}=0.029$ ) as compared with patients with lower LUS score (Fig. 1). On CT examination, significantly higher scores were measured in patients with higher LUS score (10 vs 4, p=0.027); Fig. 2 depicts LUS and CT findings in a representative patient. Rate of ace-inhibitor or angiotensin receptor blocker intake before hospital admission was similar between LUS subgroups.

\section{Analysis of Relationships}

Analysis of relationships results are summarized in Table 2 and Fig. 3. We observed a significant correlation between LUS and CT scores $(r=0.390, p=0.023)$. Both LUS and CT scores correlated directly with patients age $(\mathrm{r}=0.586, \mathrm{p}<0.001$ and $\mathrm{r}=0.399, \mathrm{p}=0.021$, respectively) as well as CRP $(\mathrm{r}=0.472$, $\mathrm{p}=0.002$ and $\mathrm{r}=0.518, \mathrm{p}=0.002$, respectively) and inversely with $\mathrm{PaO} 2 / \mathrm{FiO} 2$ ( $\mathrm{r}=-0.485, \mathrm{p}=0.003$ and $\mathrm{r}=-0.440, \mathrm{p}=0.017$ respectively). On the other hand, only LUS score had significant correlation with hs-troponin T, Nt-pro-BNP, and creatinine $(\mathrm{r}=0.433, \mathrm{p}=0.019 ; \mathrm{r}=0.411, \mathrm{p}=0.027$; and $\mathrm{r}=0.497$, $\mathrm{p}=0.001$, respectively), whereas CT score had none (all $\mathrm{p}>0.05)$.

\section{Discussion}

We reported LUS and CT findings in a cohort of patients affected by COVID-19. We observed a significant correlation between scores assessed by LUS and CT, respectively. Higher scores by either technique correlated directly with age and CRP and inversely with $\mathrm{PaO} 2 / \mathrm{FiO} 2$; however, LUS score only showed significant direct correlation with hs-troponin T, NT-pro-BNP, and creatinine.

Previous studies in COVID-19 showed that LUS semiquantitative evaluation provided useful diagnostic [14] and prognostic $[15,16]$ information, while it correlated well with worsening respiratory insufficiency [14]. In our sample, we reported similar results; however, in comparison to others [14, 15], we observed lower values of LUS scores and higher $\mathrm{PaO} 2 / \mathrm{FiO} 2$, possibly partly explained by the non-critical care setting of our population [9]. Of note, COVID-19 is featured by quite heterogeneous clinical behavior, ranging from asymptomatic cases to different degrees of flu-like symptomatology and bilateral pneumonia complicated by respiratory failure $[5,11]$. Taken together these data suggest that LUS scoring could be a reliable index of disease severity across all the COVID-19 spectrum.
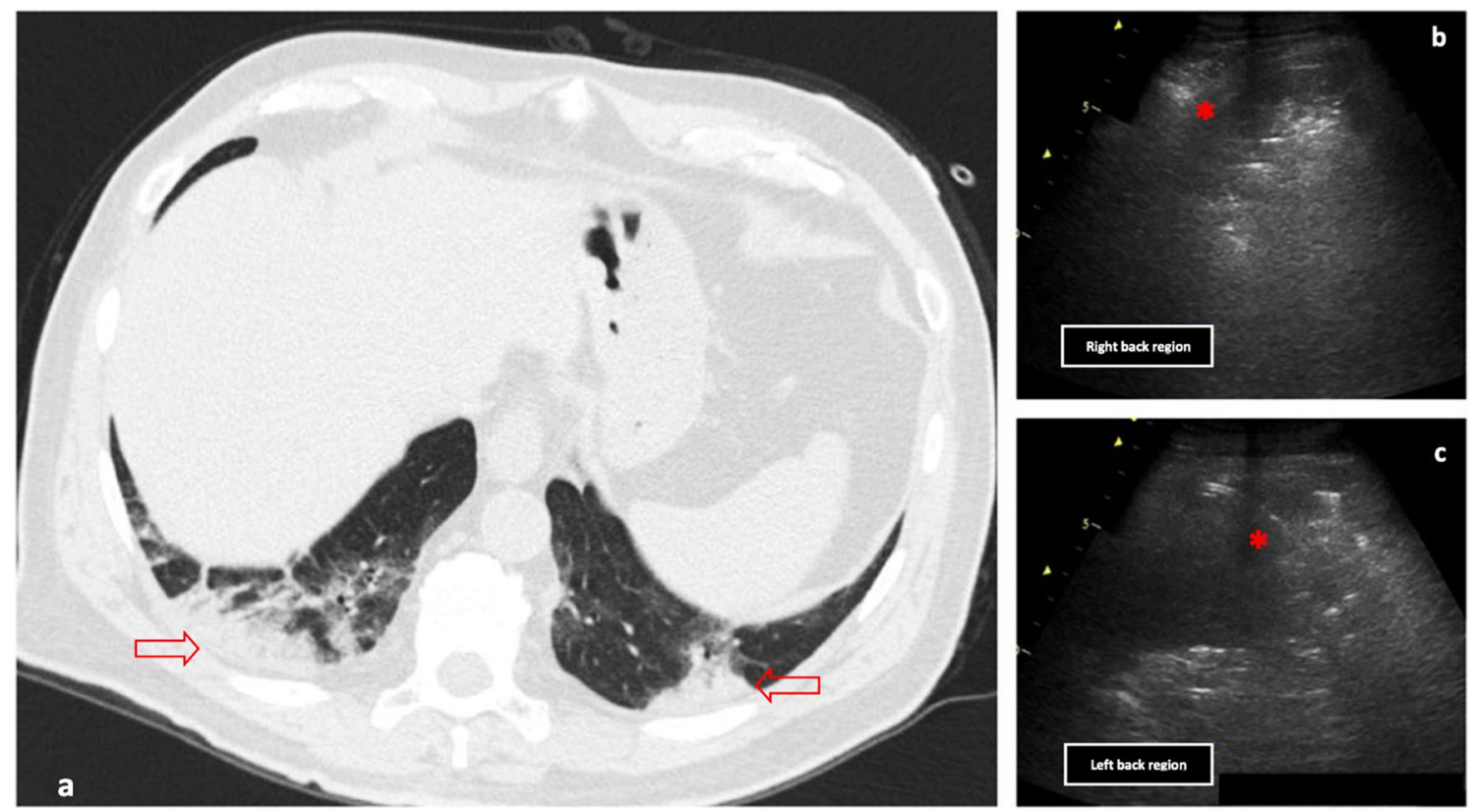

Fig. 1 Boxplot shows significantly higher CRP (A) and lower PaO2/FIO2 (B) in patient with high median LUS score 

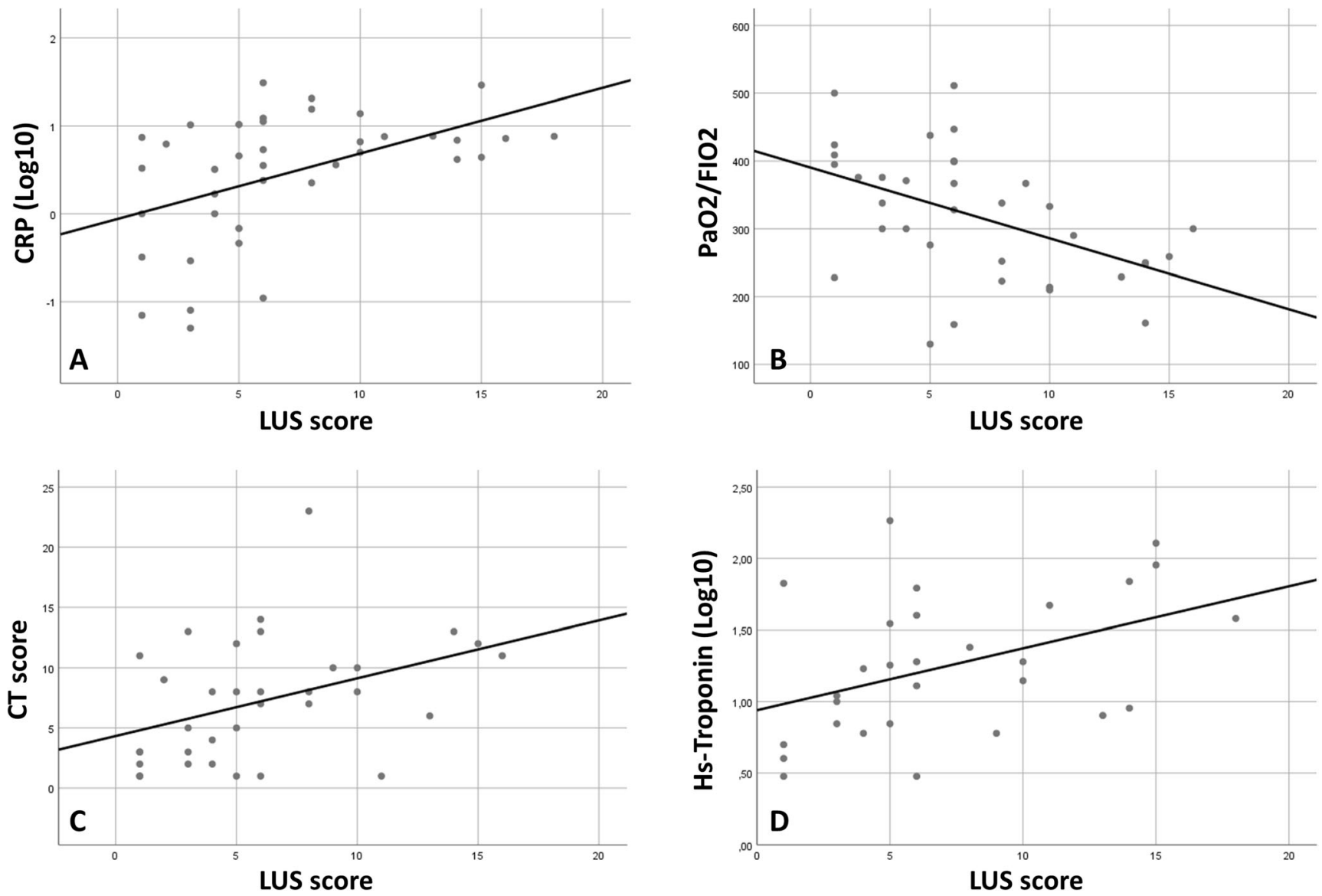

Fig. 2 Images from a 60-year-old man hospitalized for COVID-19 pneumonia. High-resolution chest computed tomography revealed focal consolidation and ground-glass pleural opacities in both inferior lobes (red

In the majority of the study population, we were able to provide comparison of LUS with chest CT results evaluated by a scoring system described in literature [12]. As already reported by others $[17,18]$, we observed significant agreement between techniques, indicating that both could be informative regarding lung and systemic involvement in these patients. Indeed, in our sample, both correlated with markers of worsening respiratory insufficiency such as $\mathrm{PaO} 2 / \mathrm{FiO} 2$ and

arrows in panel a). Lung ultrasound confirmed the presence of consolidation areas in both posterior lung lobes (red asterisk in panels $\mathbf{b}$ and $\mathbf{c}$ )

inflammation (CRP and D-dimer). Of note, LUS score only showed significant association with markers of cardiac and renal injury. Patients with COVID-19, especially those with older age included in our cohort [9], are characterized by high comorbidity burden including cardiovascular and renal diseases [19-21], which in turn can relate to pulmonary congestion through volume overload.
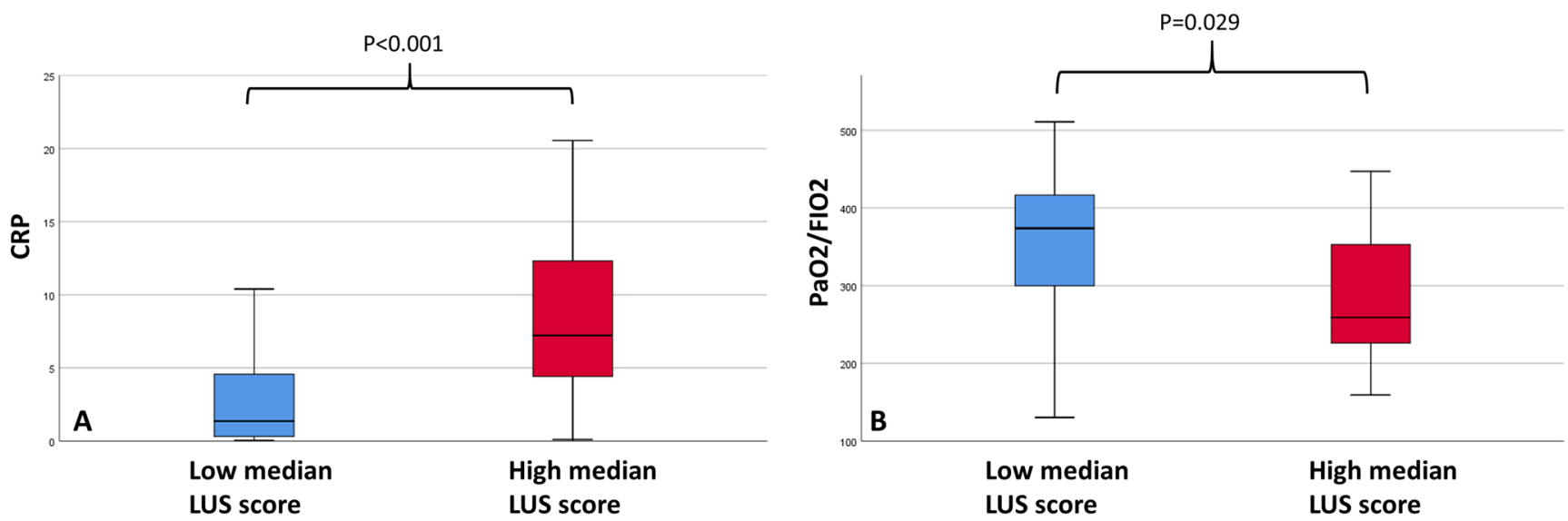

Fig. 3 Scatter plots showing correlations between LUS score and CRP (A), PaO2/FIO2 (B), CT score (C) and hs-Troponin (D), all p<0.05 
Table 2 Univariable correlation between LUS and CT score with clinical and laboratory parameters

\begin{tabular}{|c|c|c|c|c|}
\hline \multirow[t]{2}{*}{ Variable } & \multicolumn{2}{|c|}{ LUS score $(n=42)$} & \multicolumn{2}{|c|}{ CT score $(n=34)$} \\
\hline & $\mathrm{R}$ & $\mathrm{P}$ & $\mathrm{R}$ & $\mathrm{p}$ \\
\hline Age & 0.586 & $<0.001$ & 0.399 & 0.021 \\
\hline Sex (male) & -0.171 & 0.280 & -0.040 & 0.822 \\
\hline Previous CVD & 0.215 & 0.171 & 0.111 & 0.533 \\
\hline Ace-inhibitor therapy & 0.045 & 0.819 & 0.146 & 0.496 \\
\hline WBC & 0.153 & 0.347 & 0.140 & 0.436 \\
\hline Neutrophil & 0.208 & 0.198 & 0.029 & 0.873 \\
\hline Lymphocyte & -0.175 & 0.279 & 0.219 & 0.221 \\
\hline NLR & 0.241 & 0.135 & -0.113 & 0.531 \\
\hline Creatinine & 0.497 & 0.001 & 0.302 & 0.087 \\
\hline CRP $(\log 10)$ & 0.472 & 0.002 & 0.518 & 0.002 \\
\hline D-dimer $(\log 10)$ & 0.182 & 0.418 & 0.097 & 0.685 \\
\hline Hs-troponin $\mathrm{T}(\log 10)$ & 0.433 & 0.019 & 0.131 & 0.541 \\
\hline NT-pro-BNP (Log10) & 0.411 & 0.027 & 0.163 & 0.437 \\
\hline $\mathrm{PaO} 2 / \mathrm{FiO} 2$ & -0.485 & 0.003 & -0.440 & 0.017 \\
\hline CT score & 0.390 & 0.023 & NA & NA \\
\hline LUS score & NA & NA & 0.390 & 0.023 \\
\hline
\end{tabular}

LUS lung ultrasound, $C V D$ cardiovascular disease, $C R P$ C-reactive protein, $C K D$ chronic kidney disease, $C O P D$ chronic obstructive pulmonary disease, $W B C$ white blood cells, $N L R$ neutrophil to lymphocyte ratio, $\mathrm{Nt}-$ pro-BNP (), $C T$ computed tomography

Previous studies showed that LUS can be more effective than conventional chest X-ray for diagnosis of acute heart failure [22], while it can identify lung congestion in the context of volume overload driven by acute kidney injury [23, 24]. Both conditions at LUS evaluation are characterized by diffuse B-lines. Accordingly, volume overload and lung congestion might at least partly explain why degree of cardiac and renal injury were related to LUS but not CT findings in our cohort. If on one hand this result reiterates the high specificity of a semi-quantitative chest $\mathrm{CT}$ scoring system in evaluating COVID-19 lung pneumonia, on the other, it suggests that LUS might potentially provide added value in these highly comorbid patients, in which pneumonia might not be the sole cause of dyspnea and respiratory insufficiency.

Our findings might carry potential clinical implications. COVID-19 is a highly contagious disease [25], for which dedicated multidisciplinary "COVID units" have been created for the safety management of affected patients [26]. Imaging testing constitutes a potential risk for healthcare personnel, and its use should be accurately weighted to reduce the number of unnecessary examinations [27]. Our study showed that LUS could be used for pneumonia severity evaluation in COVID-19 providing results comparable to chest $\mathrm{CT}$ in the assessment of lung and systemic inflammatory involvement, with the added advantage of being effectively executable bedside.

\section{Limitations}

Our study should be read in light of several limitations, such as partly retrospective nature, limited sample size, and noncritical care setting of care which can reduce generalizability of our findings. The associations we observed between imaging scoring and systemic COVID-19 involvement must be cautiously interpreted as hypothesis generating only, not allowing to draw patterns of existing cause-effect relationships. Larger prospective studies are needed to fully assess whether LUS could be reliably used as possible alternative to chest CT scan for the diagnosis of COVID-19 and inhospital monitor of disease evolution.

\section{Conclusions}

In patients with COVID-19 pneumonia admitted to noncritical care wards, semi-quantitative bedside LUS evaluation identified the subgroup of patients with worse respiratory failure and systemic inflammation with results comparable to chest CT evaluation. Correlation of LUS score with markers of cardiac and renal injury suggests that LUS might contribute to a more comprehensive evaluation, potentially aiding detection of non-pneumonia-related causes of respiratory insufficiency within this heterogeneous and highly comorbid population.

Author Contribution Performed imaging tests (GP, FC, MD, SM), involved in the care of the patients (all authors), literature search (GP, FC, LA), wrote the draft of the manuscript (GP, FC, LA, MD), and provided critical revision and approved the final version of the manuscript (all authors)

Data Availability Data will be made available upon request.

Code Availability Not applicable

\section{Declarations}

Ethics Approval Institutional review board approved the study.

Consent to Participate The patients provided written informed consent to the used of data for research purpose.

Consent for Publication The patients consented to the publication of anonymized data.

Conflict of Interest The authors declare no competing interests. 


\section{References}

1. Zhu N, Zhang D, Wang W, Li X, Yang B, Song J, et al. A novel coronavirus from patients with pneumonia in China, 2019. N Engl J Med. 2020;382:727-33. https://doi.org/10.1056/NEJMoa2001017.

2. Puntmann VO, Carerj ML, Wieters I, Fahim M, Arendt C, Hoffmann J, et al. Outcomes of cardiovascular magnetic resonance imaging in patients recently recovered from coronavirus disease 2019 (COVID-19). JAMA Cardiol. 2020;5:1265-73. https://doi. org/10.1001/jamacardio.2020.3557.

3. Camastra G, Ciolina F, Arcari L, et al. Heart and lung involvement detected by native $\mathrm{T} 1$ and $\mathrm{T} 2$ mapping magnetic resonance imaging in a patient with coronavirus disease-19. Eur Heart J Cardiovasc Imaging. 2021. https://doi.org/10.1093/ehjci/jeaa414.

4. Choudry FA, Hamshere SM, Rathod KS, Akhtar MM, Archbold RA, Guttmann OP, et al. High thrombus burden in patients with COVID-19 presenting with ST-segment elevation myocardial infarction. J Am Coll Cardiol. 2020;76:1168-76. https://doi.org/10. 1016/j.jacc.2020.07.022.

5. Huang C, Wang Y, Li X, Ren L, Zhao J, Hu Y, et al. Clinical features of patients infected with 2019 novel coronavirus in Wuhan, China. Lancet. 2020;395:497-506. https://doi.org/10. 1016/S0140-6736(20)30183-5.

6. Larici AR, Cicchetti G, Marano R, Merlino B, Elia L, Calandriello $\mathrm{L}$, et al. Multimodality imaging of COVID-19 pneumonia: from diagnosis to follow-up. A comprehensive review. Eur J Radiol. 2020;131:109217.

7. Harmon SA, Sanford TH, Xu S, Turkbey EB, Roth H, Xu Z, et al. Artificial intelligence for the detection of COVID-19 pneumonia on chest CT using multinational datasets. Nat Commun. 2020;11:1-7. https://doi.org/10.1038/s41467-020-17971-2.

8. Sultan LR, Sehgal CM. A review of early experience in lung ultrasound in the diagnosis and management of COVID-19. Ultrasound Med Biol. 2020;46:2530-45. https://doi.org/10.1016/j. ultrasmedbio.2020.05.012.

9. Arcari L, Luciani M, Cacciotti L, Musumeci MB, Spuntarelli V, Pistella E, et al. Incidence and determinants of high-sensitivity troponin and natriuretic peptides elevation at admission in hospitalized COVID-19 pneumonia patients. Intern Emerg Med. 2020;15:146776. https://doi.org/10.1007/s11739-020-02498-7.

10. Salehi S, Abedi A, Balakrishnan S, Gholamrezanezhad A. Coronavirus disease 2019 (COVID-19): a systematic review of imaging findings in 919 patients. Am J Roentgenol. 2020;215: 87-93.

11. Ye Z, Zhang Y, Wang Y, Huang Z, Song B. Chest CT manifestations of new coronavirus disease 2019 (COVID-19): a pictorial review. Eur Radiol. 2020;30:4381-9. https://doi.org/10.1007/ s00330-020-06801-0.

12. Pan F, Ye T, Sun P, Gui S, Liang B, Li L, et al. Time course of lung changes at chest CT during recovery from Coronavirus disease 2019 (COVID-19). Radiology. 2020;295:715-21. https://doi.org/ 10.1148/radiol.2020200370.

13. Soldati G, Smargiassi A, Inchingolo R, Buonsenso D, Perrone T, Briganti DF, et al. Proposal for international standardization of the use of lung ultrasound for COVID-19 patients; a simple, quantitative, reproducible method. J Ultrasound Med. 2020;39:1413-9. https://doi.org/10.1002/jum.15285.

14. Bosso G, Allegorico E, Pagano A, Porta G, Serra C, Minerva V, et al. Lung ultrasound as diagnostic tool for SARS-CoV-2 infection. Intern Emerg Med. 2020;16:471-6. https://doi.org/10.1007/ s11739-020-02512-y.

15. Lichter Y, Topilsky Y, Taieb P, Banai A, Hochstadt A, Merdler I, et al. Lung ultrasound predicts clinical course and outcomes in
COVID-19 patients. Intensive Care Med. 2020;46:1873-83. https://doi.org/10.1007/s00134-020-06212-1.

16. Rojatti M, Regli IB, Zanforlin A, Ferretti E, Falk M, Strapazzon G, et al. Lung ultrasound and respiratory pathophysiology in mechanically ventilated COVID-19 patients - an observational trial. SN Compr Clin Med. 2020;2:1970-7. https://doi.org/10.1007/s42399020-00536-1.

17. Tung-Chen Y, Martí de Gracia M, Díez-Tascón A, AlonsoGonzález R, Agudo-Fernández S, Parra-Gordo ML, et al. Correlation between chest computed tomography and lung ultrasonography in patients with coronavirus disease 2019 (COVID-19). Ultrasound Med Biol. 2020;46:2918-26. https://doi.org/10.1016/j. ultrasmedbio.2020.07.003.

18. Marggrander DT, Borgans F, Jacobi V, Neb H, Wolf T. Lung ultrasound findings in patients with COVID-19. SN Compr Clin Med. 2020;2:2151-7. https://doi.org/10.1007/s42399-020-005530 .

19. Núñez-Gil IJJ, Fernández-Ortiz A, Maroud Eid C, et al. Underlying heart diseases and acute COVID-19 outcomes. Cardiol J. 2020;28: 202-14. https://doi.org/10.5603/cj.a2020.0183.

20. Liu Y-F, Zhang Z, Pan X-L, Xing GL, Zhang Y, Liu ZS, et al. The chronic kidney disease and acute kidney injury involvement in COVID-19 pandemic: a systematic review and meta-analysis. PLoS One. 2021;16:e0244779. https://doi.org/10.1371/journal. pone. 0244779 .

21. Uribarri A, Núñez-Gil IJ, Aparisi A, et al. Impact of renal function on admission in COVID-19 patients: an analysis of the international HOPE COVID-19 (Health Outcome Predictive Evaluation for COVID 19) Registry. J Nephrol. 2020;33:737-45. https://doi.org/ 10.1007/s40620-020-00790-5.

22. Rinaldi L, Milione S, Fascione MC, Pafundi PC, Altruda C, di Caterino M, et al. Relevance of lung ultrasound in the diagnostic algorithm of respiratory diseases in a real-life setting: a multicentre prospective study. Respirology. 2020;25:535-42. https://doi.org/ 10.1111/resp.13659.

23. Ciumanghel A, Siriopol I, Blaj M, Siriopol D, Gavrilovici C, Covic A. B-lines score on lung ultrasound as a direct measure of respiratory dysfunction in ICU patients with acute kidney injury. Int Urol Nephrol. 2018;50:113-9. https://doi.org/10.1007/s11255-0171730-8.

24. Panuccio V, Tripepi R, Parlongo G, Mafrica A, Caridi G, Catalano $\mathrm{F}$, et al. Lung ultrasound to detect and monitor pulmonary congestion in patients with acute kidney injury in nephrology wards: a pilot study. J Nephrol. 2020;33:335-41. https://doi.org/10.1007/ s40620-019-00666-3.

25. Li Q, Guan X, Wu P, Wang X, Zhou L, Tong Y, et al. Early transmission dynamics in Wuhan, China, of novel coronavirusinfected pneumonia. N Engl J Med. 2020;382:1199-207. https:// doi.org/10.1056/NEJMoa2001316.

26. Pennica A, Conforti G, Falangone F, Martocchia A, Tafaro L, Sentimentale A, et al. Clinical management of adult coronavirus infection disease 2019 (COVID-19) positive in the setting of low and medium intensity of care: a short practical review. SN Compr Clin Med. 2020;2:1-6. https://doi.org/10.1007/s42399-020-00333w.

27. Skulstad H, Cosyns B, Popescu BA, et al. COVID-19 pandemic and cardiac imaging: EACVI recommendations on precautions, indications, prioritization, and protection for patients and healthcare personnel. https://doi.org/10.1093/ehjci/jeaa072.

Publisher's Note Springer Nature remains neutral with regard to jurisdictional claims in published maps and institutional affiliations. 